Amir Rahimirad ${ }^{1}$, Afshin Javadi ${ }^{1, \star}$, Hamid Mirzaei ${ }^{1}$, Navideh Anarjan ${ }^{2}$ and Hoda Jafarizadeh-Malmiri ${ }^{3}$

\title{
Biosynthetic potential assessment of four food pathogenic bacteria in hydrothermally silver nanoparticles fabrication
}

https://doi.org/10.1515/gps-2019-0033

Received November 23, 2018; accepted April 29, 2019.

Abstract: Silver nanoparticles (Ag NPs) were synthesized using four pathogenic bacterial extracts namely, Bacillus cereus, E. coli, Staphylococcus aureus and Salmonella entericasubsp.enterica. Synthesis process were hydrothermally accelerated using temperature, pressure and heating time of $121^{\circ} \mathrm{C}, 1.5$ bar ad $15 \mathrm{~min}$. Physico-chemical characteristics of the fabricated Ag NPs, including, particle size, polydispersity index (PDI), zeta potential, broad emission peak $\left(\lambda_{\max }\right)$ and concentration were evaluated using UV-Vis spectrophotometer and dynamic light scattering (DLS) particle size analyzer. Furthermore, main existed functional groups in the provided bacterial extracts were recognized using Fourier transform infrared spectroscopy. The obtained results revealed that two main peaks were detected around 3453 and $1636.5 \mathrm{~cm}^{-1}$, for all bacterial extracts, were interrelated to the stretching vibrations of hydroxyl and amide groups which those had key roles in the reduction of ions and stabilizing of the formed Ag NPs. The results also indicated that, Ag NPs with much desirable characteristics, including minimum particle size $(25.62 \mathrm{~nm})$ and PDI (0.381), and maximum zeta potential $(-29.5 \mathrm{mV})$ were synthesized using S. e. subsp. enterica extract. $\lambda_{\max }$, absorbance and concentration values for the fabricated Ag NPs with this bacterial extract were $400 \mathrm{~nm}$, $0.202 \%$ a.u. and $5.87 \mathrm{ppm}$.

Keywords: silver nanoparticles; biosynthesis; pathogens; bacteria strains; physico-chemical properties

\footnotetext{
* Corresponding author: Afshin Javadi, Department of Food Hygiene, Tabriz Branch, Islamic Azad University, Tabriz, Iran,

Tel.: +98 9141165059, Fax: +984136376934

e-mail: Javadi@iaut.ac.ir

Amir Rahimirad and Hamid Mirzaei, Department of Food Hygiene, Tabriz Branch, Islamic Azad University, Tabriz, Iran

Navideh Anarjan, Faculty of Chemical Engineering, Tabriz Branch, Islamic Azad University, Tabriz, Iran

Hoda Jafarizadeh-Malmiri, Faculty of Chemical Engineering, Sahand University of Technology, 51335-1996 Sahand, East Azarbaijan, Tabriz, Iran
}

\section{Introduction}

Among metal and metal oxide nanoparticles, silver nanoparticles (Ag NPs) have attained more attention and interests due to their unique attributes, specially their enormous antimicrobial activity. As compared to bulk form of silver, Ag NPs have high surface to volume ratio which in turn, increases the surface energy of the Ag NPs to easily attach into the microorganisms cytoplasmic membrane and change their permeability and cause their death [1]. Furthermore, several studies indicated that there are another two mechanisms related to antimicrobial activity of Ag NPs, including inactivation of respiratory enzymes of mitochondria (near to the cell membrane) and DNA replication disruption [2,3]. Massive antimicrobial activity of Ag NPs against numerous microorganisms such as bacteria and fungi strains has developed their applications in various industries and fields including, food, biotechnology, waste water treatment, tissue engineering, paint, electronic devices, automobile and medicines [4,5].

Biogenic synthesis of metal NPs using microorganism is a novel branch of the nanotechnology which is known as nanobiotechnology and deals with great attention by the researchers, these days. In fact, nanobiotechnology is an emerging field of research at the crossroads of biotechnology and nanoscience which by intersection of inorganic and organic engineering solves critical problems in biology [1,6]. Presence of numerous biomolecules in the microorganisms, such as proteins, polysaccharides, lipids, nucleic acids and enzymes, make those attractive to reduce metal ions and convert them into the NPs, and stabilize the formed NPs. It has been revealed that, these biomolecules could be effectively used in the biosynthesis of inorganic NPs, as reducing and stabilizing agents $[7,8]$. Several studies have been done on biosynthesis of Ag NPs using different fungi (e.g. Aspergillus fumigatus) and bacteria (e.g. E. coli, Pseudomonas aeruginosa and Staphylococcus aureus) strains [9,10].

There are two approaches in biological synthesis of metal NPs using microorganisms namely, intracellular and 
extracellular [10]. In the intracellular method the metal salt, as ion source, is added into the broth culture media and after that provided microbe strain complemented to that. During incubation of the media, microorganisms growth and absorb the ions, and synthesis metal NPs in themselves. However, the extracellular synthesis can be implemented not only using some microorganisms whole cells, but also it is applicable employing cell filtrate, lysate, supernatant and cellular components of the microbe [1]. Due to antimicrobial activities of some metal ions and their inhibitory effects on the growth of microorganisms, extracellular approach has been preferred [9].

Some of the microorganisms are pathogens and should be remove from the food and environment. Using these useless microorganisms to synthesis of metal NPs, such as Ag NPs, which those have antimicrobial activity and can be utilized in various areas, is valuable and more attractive subject during last years. Therefore, the main objectives of the present study were to i) evaluate the Ag NPs synthetic potential of four food pathogens bacteria namely, Bacillus cereus, E. coli, Staphylococcus aureus and Salmonella entericasubsp. enterica and ii) assess physicochemical properties of the fabricated Ag NPs including, concentration, particle size, polydispersity index and zeta potential values.

\section{Materials and methods}

\subsection{Materials}

Silver nitrate (AgNO3) was purchased from Merck (Merck Co., Darmstadt, Germany). Standard Ag NPs colloid solution, with concentration of $1000 \mathrm{ppm}$ and particle size of $10 \mathrm{~nm}$, was provided from Tecnan-Nanomat Co. (Navarra, Spain). Bacillus cereus (PTCC 1015), E. coli (PTCC 1276), Staphylococcus aureus (PTCC 1431) and Salmonella entericasubsp. enterica (PTCC 1787) were obtained from microbial Persian type culture collection (PTCC, Tehran, Iran). Specifications of these four bacteria strains show in Table 1. Tryptic soy (CASO) broth, tryptic soy agar and nutrient agar were bought from Merck (Merck Co., Darmstadt, Germany).

\subsection{Preparation of bacterial extract}

In order to preparation of the bacterial biomass, after culturing the provided bacteria strains on the surface of the plates containing nutrient agar (for E. coli, B. cereus and S. e. subsp. enterica) and tryptic soy agar
Table 1: Specifications of four studied bacteria strains.

\begin{tabular}{|c|c|c|c|c|}
\hline $\begin{array}{l}\text { Bacteria } \\
\text { strain }\end{array}$ & $\begin{array}{l}\text { Gram } \\
+/-\end{array}$ & Need to $\mathrm{O}_{2}$ & Shape & $\begin{array}{r}\text { Optimum } \\
\text { temperature } \\
\text { to growth }\left({ }^{\circ} \mathrm{C}\right)\end{array}$ \\
\hline E. coli & - & Facultative anaerobic & Rod & 37 \\
\hline S. aureus & + & Facultative anaerobic & Spherical & 37 \\
\hline B. cereus & + & $\begin{array}{r}\text { Aerobic/ Facultative } \\
\text { anaerobic }\end{array}$ & Rod & 30 \\
\hline $\begin{array}{l}\text { S. e. subsp. } \\
\text { enterica }\end{array}$ & - & Facultative aerobic & Rod & 37 \\
\hline
\end{tabular}

(for S. aureus) the plates were incubated at $37^{\circ} \mathrm{C}$ for $48 \mathrm{~h}$ excepted those containing $B$. cereus. The plates those were surfaced cultured with this bacteria strain were incubated at $30^{\circ} \mathrm{C}$ for $48 \mathrm{~h}$. After that, all separated colonies of each bacterium strains were added into $40 \mathrm{~mL}$ of CASO broth and incubated in a laboratory incubator (M30, Memmert GmbH \& Co.KG, Schwa Bach, Germany) adjusted at two different temperatures $\left(30\right.$ and $37^{\circ} \mathrm{C}$ ) for two days, as already has explained. After that, using a laboratory centrifuge (Microo 220 R, Andreas Hettich GmbH \& Co.KG, Tuttlingen, Germany) adjusted at $6000 \mathrm{rpm}$ at $25^{\circ} \mathrm{C}$ for $10 \mathrm{~min}$, the bacterial cells were separated and washed again using deionized distilled water (DDW) and centrifuged. Finally the provided biomass was added into $50 \mathrm{~mL}$ of DDW and kept in a refrigerated incubator (KB115, $\mathrm{GmbH} \&$ Co.KG, Tuttlingen, Germany) for $48 \mathrm{~h}$ to cell disintegration. By centrifugation of the samples, bacterial cell free extract were separated from the cell debris and kept in the refrigerator $\left(4^{\circ} \mathrm{C}\right)$ throughout the experiments.

\subsection{Synthesis of Ag NPs using bacterial extract}

Based on the most literatures, a $1 \mathrm{mM}$ silver nitrate was prepared by dissolving of $0.017 \mathrm{~g}$ of $\mathrm{AgNO}_{3}$ into the $100 \mathrm{~mL}$ DDW, as silver ions precursor [4,9,11]. After that, $1 \mathrm{~mL}$ of the silver salt solution was then added into the $3 \mathrm{~mL}$ of each bacterial extracts and the mixture solutions were placed in a laboratory autoclave (AM A240T, Astell Co., Sidcup, UK) adjusted at $121^{\circ} \mathrm{C}$ and 1.5 bar (pressure) for $15 \mathrm{~min}$.

\subsection{Physico-chemical analysis}

\subsubsection{Bacterial extracts}

Fourier transform infrared (FT-IR) spectra can easily detect and indicate the main functional groups presented in the bacterial extracts. These functional groups, based 
on the literatures, can reveal the presence of some biomolecules such as proteins and polysaccharides in the extracts, which those act as reducing and stabilizing agents in the formation NPs [12,13]. For this reason, bacterial extracts were monitored in $\mathrm{KBr}$ pellets using a FT-IR spectrophotometer (Shimadzu 8400S, Shimadzu Co., Kyoto, Japan) adjusted at range of $4000-400 \mathrm{~cm}^{-1}$.

\subsubsection{Synthesized Ag NPs}

Ag NPs due to their surface Plasmon resonance (SPR) have a broad emission peak $\left(\lambda_{\max }\right)$ placed at wavelength of $380-450 \mathrm{~nm}$, which this peak easily confirmed the formation of Ag NPs [2,9]. Therefore, using a UV-Vis spectrophotometer (UV-1800, Shimadzu Co., Tokyo, Japan) and scanning of the mixture solutions after hydrothermal process, fabrication of the Ag NPs using four different bacterial extracts were evaluated.

Furthermore, it is possible to calculate the concentration of the formed Ag NPs using UV-Vis spectroscopy. For this reason, a standard curve, using serial dilute solutions of the provided standard AgNPs (1-10 ppm) was prepared which in that, the absorbance (\% a.u.) of the fabricated Ag NPs was correlated to their concentration.

Dynamic light scattering (DLS) particle size analyzer (Nanotrac Wave, Microtrac, USA) was also utilized to measure particle size, polydispersity index (PDI) and Zeta potential values and to monitor particle size distribution (PSD) of the formed Ag NPs.

\subsection{Experimental design and statistical analysis}

Experiments were design based on full factorial and all analysis related to the characteristics of the fabricated Ag NPs were completed in three replications. Analysis of variance (ANOVA) using Minitab v.16 statistical package (Minitab Inc., PA, USA) was used to statistical analysis. Tuky's comparison test was also used to compare the mean values, at $5 \%$ level of significance.

\section{Results and discussions}

\subsection{FT-IR spectra of the bacterial extracts}

Figure 1 shows FT-IR spectra of the four prepared bacterial extracts. As can be seen in this figure, there are

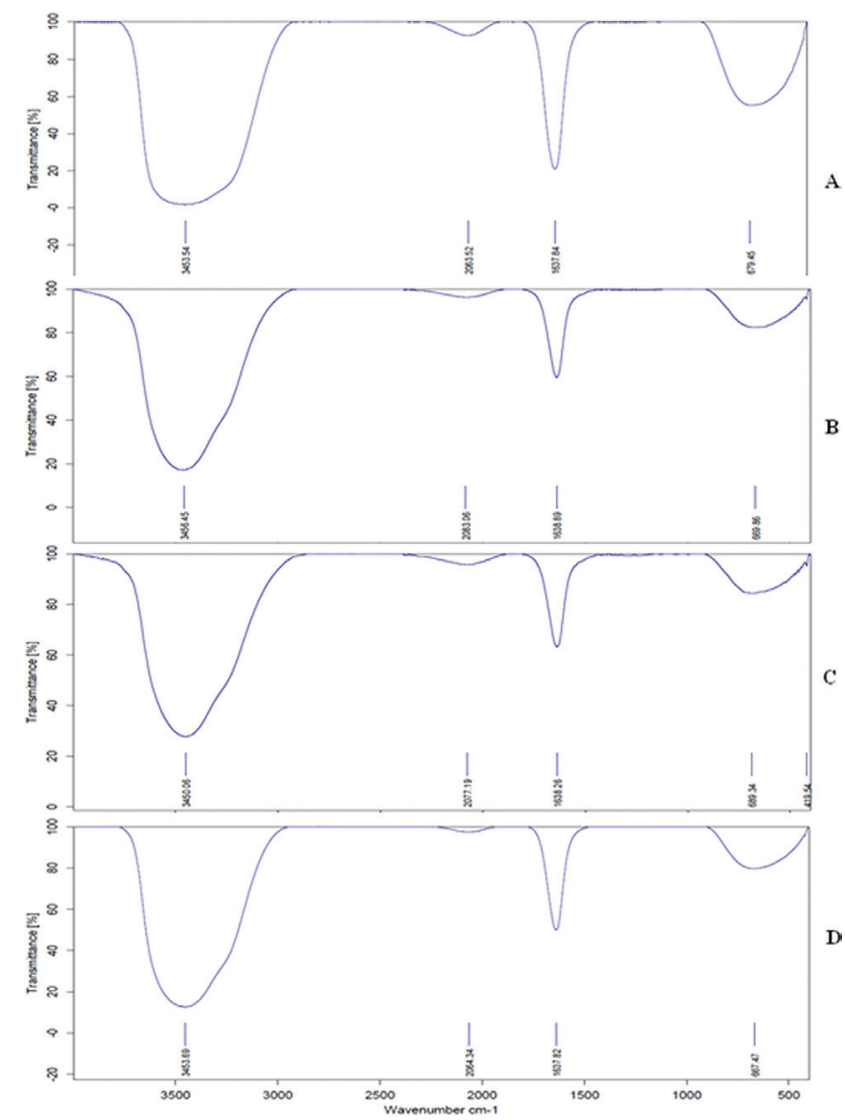

Figure 1: FT-IR spectrum of E. coli (a), S. aureus (b), B. cereus (c) and S. e. subsp. enterica (d) extracts.

4 dominated peaks for all the extracts which those are centered at 3450-3456, 2063-2083, 1637 and $667-689 \mathrm{~cm}^{-1}$. The two main peaks were detected around 3453 and $1636.5 \mathrm{~cm}^{-1}$, for all bacterial extracts, were interrelated to the stretching vibrations of hydroxyl and amide groups, respectively. Hydroxyl group which is the main functional group of the polysaccharides, nucleic acids and other main components existed in the bacterial cell has main role in reduction of silver ions and finally synthesis of $\mathrm{Ag}$ NPs [5]. Amide group is also the main functional group which is presented in proteins and enzymes and has a key role in stabilizing of the fabricated Ag NPs [9]. The obtained results revealed that all four selected bacteria strains had synthetic and stabilizing potentials to fabricated stable Ag NPs.

\subsection{SPR and concentration of the fabricated Ag NPs}

Due to SPR character of the metal NPs which is related to the combined vibration in resonance between free electrons and the light wave, fabricated Ag NPs had broad 
emission peaks $\left(\lambda_{\max }\right)$ were located at wavelength ranging $380-450 \mathrm{~nm}[1,3,11]$. There is a direct relation between peak height and concentration of the synthesized $\mathrm{Ag}$ NPs [5]. According to the provided standard curve, using serial dilute solutions of the provided standard Ag NPs (1-10 ppm), the following equation (Eq. 1) was generated:

$$
C=32.775 X-0.7474
$$

where, $\mathrm{c}$ is the concentration of the formed Ag NPs in the colloidal solution and $\mathrm{X}$ is the absorbance of the solution at $\lambda_{\max }$. Table 2 shows the values of $\lambda_{\max }$, absorbance and concentration of the fabricated Ag NPs using different four bacterial extracts. As clearly observed in Table 2, the Ag NPs with highest concentration were synthesized using E. coli extracts. Figure 2 indicates UV-Vis spectra of the formed Ag NPs using $E$. coli extract.

\subsection{Particle size, PDI and zeta potential of the synthesized Ag NPs}

Physico-chemical characteristics of the synthesized Ag NPs using four different bacterial extracts show in Table 3.

Table 2: $\lambda_{\max }$, absorbance and concentration of the fabricated Ag NPs using bacterial extracts.

\begin{tabular}{lcrr}
\hline Bacteria strain & $\begin{array}{r}\lambda_{\max } \\
(\mathbf{n m})\end{array}$ & $\begin{array}{r}\text { Absorbance } \\
(\% \text { a.u. })\end{array}$ & $\begin{array}{r}\text { Concentration } \\
(\mathbf{p p m})\end{array}$ \\
\hline E. coli & 428 & 0.253 & 7.55 \\
S. aureus & 400 & 0.086 & 2.07 \\
B. cereus & 450 & 0.103 & 2.63 \\
S. e. subsp. enterica & 400 & 0.202 & 5.87 \\
\hline
\end{tabular}

Data are mean values of three replications.

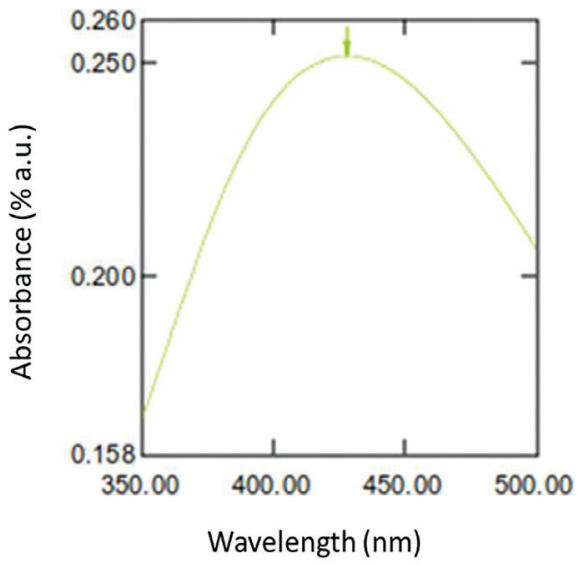

Figure 2: Surface Plasmon resonance spectrum of the synthesized Ag NPs using E. coli.
The obtained results indicated that Ag NPs with much desirable characteristics including minimum particle size $(25.62 \mathrm{~nm})$ and PDI (0.381), and maximum zeta potential $(-29.5 \mathrm{mV})$ were synthesized using S. e. subsp. enterica extract. Silambarasan and Abraham synthesized exteracellulary Ag NPs using B. cereus with particle size

Table 3: Physico-chemical characteristics of the fabricated Ag NPs using bacterial extracts.

\begin{tabular}{lrcr}
\hline Bacteria strain & $\begin{array}{r}\text { Particle size } \\
(\mathrm{nm})\end{array}$ & PDI & $\begin{array}{r}\text { Zeta potential } \\
(\mathrm{mV})\end{array}$ \\
\hline E. coli & 84.05 & 0.564 & -15.1 \\
S. aureus & 57.28 & 0.510 & -17.2 \\
B. cereus & 94.64 & 0.658 & -18.7 \\
S. e. subsp. enterica & 25.62 & 0.381 & -29.5 \\
\hline
\end{tabular}

Data are mean values of three replications.
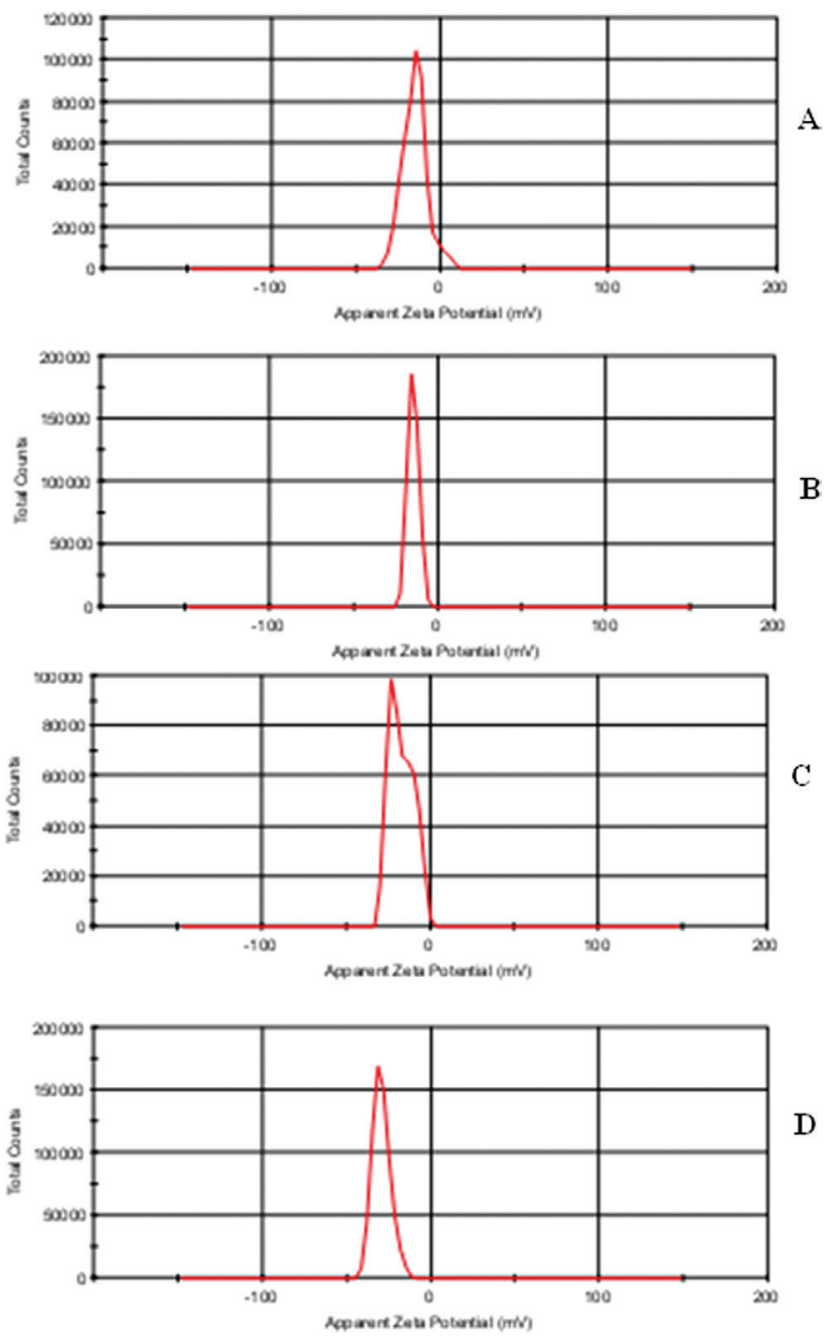

Figure 3: Zeta potential distribution of the synthesized Ag NPs using E. coli (a), S. aureus (b), B. cereus (c) and S. e. subsp. enterica (d) extracts. 
of $62.8 \mathrm{~nm}$ and $\lambda_{\max }$ of $440 \mathrm{~nm}$ [14]. Shah et al. fabricated Ag NPs using E. coli extract wiyh particle size and zeta potential values of $297.7 \mathrm{~nm}$ and $-12.4 \mathrm{mV}$, respectively [15]. Nanda and Saravanan exteracellulary synthesized Ag NPs using S. aureus with mean particle size of $170 \mathrm{~nm}$ and $\lambda_{\max }$ of $420 \mathrm{~nm}$ [16].

Obtained results also indicated small value for the PDI of the synthesized Ag NPs using S. e. subsp. enterica extract which in turn revealed that the monodispersed Ag NPs were formed. The obtained result was in line with finding of Mohammadlou et al. [4]. They fabricated monodispersed Ag NPs using Pelargonium leaf extract while the PDI of the fabricated NPs was 0.413. Furthermore, higher value of zeta potential for the fabricated Ag NPs with this bacteria strain illustrated their highest stability in the colloidal solution. This result was in agreement with achievement of Torabfam and Jafarizadeh-Malmiri [3]. They fabricated much more stable

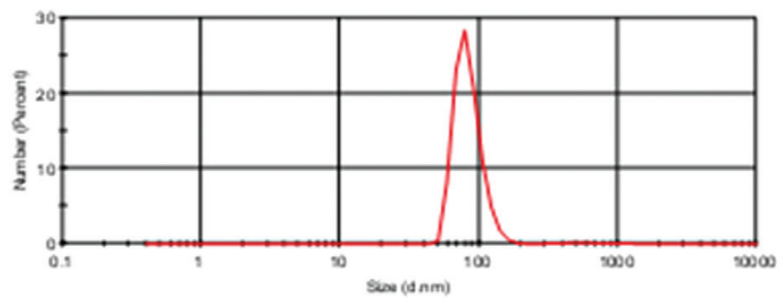

A
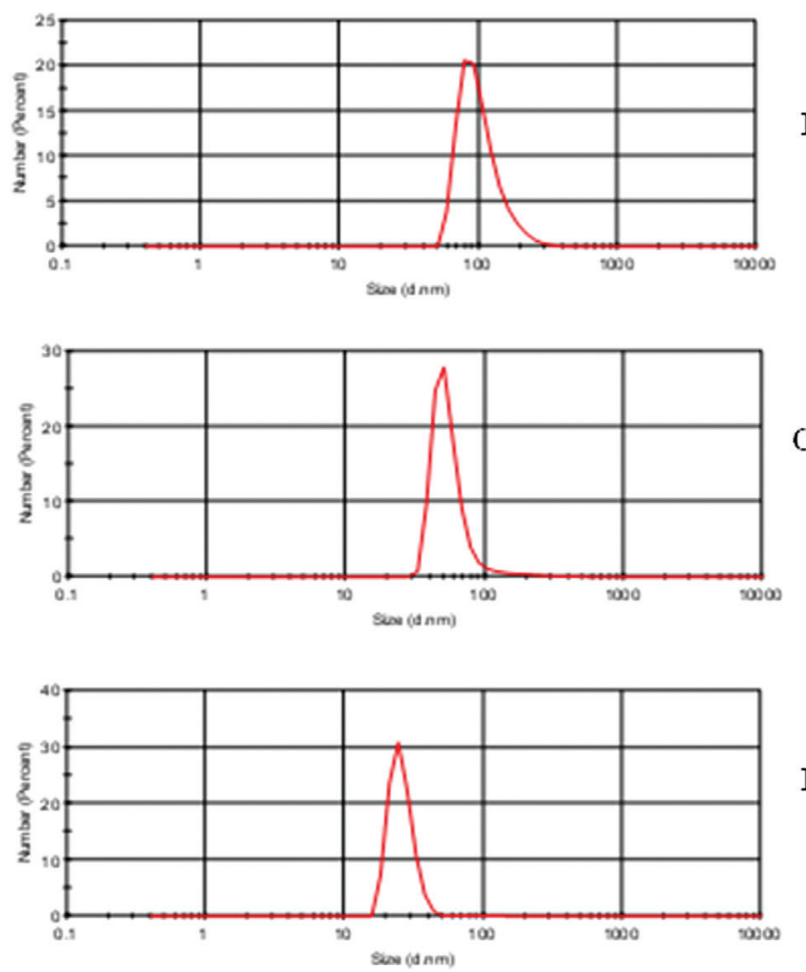

Figure 4: Particle size distribution of the synthesized Ag NPs using E. coli (a), S. aureus (b), B. cereus (c) and S. e. subsp. enterica (d) extracts.

D
Ag NPs using chitosan with zeta potential of $+50 \mathrm{mV}$. Figure 3 shows the zeta potential distribution of the synthesized Ag NPs using four different bacterial extracts.

Particle size distribution (PSD) of the synthesized Ag NPs using E. coli, S. aureus, B. cereus and S. e. subsp. enterica extracts show in Figures $4 \mathrm{a}, 4 \mathrm{~b}, 4 \mathrm{c}$, and $4 \mathrm{~d}$, respectively. The presence of sharp and narrow peaks for PSD of all four groups of the fabricated Ag NPs using different bacteria revealed that the formed Ag NPs were monodisperesed. The results were reconfirmed by the small values of the PDI for the synthesized Ag NPs with all selected bacteria strain extracts, as can be seen in Table 3 .

\section{Conclusions}

Biological synthesis of Ag NPs using bacteria strains, especially pathogens, is a boon for advance research in nanobiotechnology. However, pathogens with toxin production must be removed from the food products and killed, but, those have great potential in metal NPs synthesis, such as Ag NPs which those have strong antibacterial activity against vast microorganisms, especially the pathogens. The obtained results revealed that four selected pathogens in the present study namely, E. coli, S. aureus, B. cereus and S. e. subsp. enterica had appropriate synthetic potential for extracellular fabrication of monodispersed and stable Ag NPs with small particle size. Using pathogens in Ag NPs synthesis, according to the developed manner in the present study can be used widely in the synthesis of other noble metal and metal oxide NPs.

Acknowledgments: The authors appreciate the supports of Islamic Azad University-Tabriz branch to accomplish this research.

Conflicts of interest: All authors declare no conflict of interest.

\section{References}

[1] Mohammadlou M., Maghsoudi H., Jafarizadeh-Malmiri H., A review on green silver nanoparticles based on plants: Synthesis, potential applications and eco-friendly approach. Int. Food Res. J., 2016, 23, 446-463.

[2] Eshghi M., Vaghari H., Najian Y., Najian M.J., Jafarizadeh-Malmiri H., Berenjian A., Microwave-assisted green synthesis of silver nanoparticles using Juglans regia leaf extract and evaluation of 
their physico-chemical and antibacterial properties. Antibiotics, 2018, 7, 68-77.

[3] Torabfam M., Jafarizadeh-Malmiri H., Microwave-enhanced silver nanoparticle synthesis using chitosan biopolymer: optimization of the process conditions and evaluation of their characteristics. Green Process. Synth., 2018, 7, 530-537.

[4] Mohammadlou M., Jafarizadeh-Malmiri H., Maghsoudi H., Hydrothermal green synthesis of silver nanoparticles using Pelargonium/Geranium leaf extract and evaluation of their antifungal activity. Green Process. Synth., 2017, 6, 31-42.

[5] Ahmadi O., Jafarizadeh-Malmiri H., Jodeiri N., Optimization of processing parameters for hydrothermal silver nanoparticles synthesis using Aloe vera leaf extract and estimation of their physico-chemical and antifungal properties. Z. Phys. Chem. (in press), DOI:10.1515/zpch-2017-1089.

[6] Sekhon B.S., Nanotechnology in agri-food production: an overview. Nanotechnol. Sci. Appl., 2014, 7, 31-53.

[7] Eskandari-Nojehdehi M., Jafarizadeh-Malmiri H., RahbarShahrouzi J., Optimization of processing parameters in green synthesis of gold nanoparticles using microwave and edible mushroom (Agaricus bisporus) extract and evaluation of their antibacterial activity. Nanotechnol. Rev., 2016, 5, 537-548.

[8] Eskandari-Nojedehi M., Jafarizadeh-Malmiri H., RahbarShahrouzi J., Hydrothermal green synthesis of gold nanoparticles using mushroom (Agaricus bisporus) extract: physico-chemical characteristics and antifungal activity studies. Green Process. Synth., 2018, 7, 38.

[9] Ghanbari S., Vaghari H., Sayyar Z., Adibpour M., JafarizadehMalmiri H., Autoclave-assisted green synthesis of silver nanoparticles using $A$. fumigatus mycelia extract and the evaluation of their physico-chemical properties and antibacterial activity. Green Process. Synth., 2018, 7, 217-224.

[10] Birla S.S., Tiwari V.V., Gade A.K., Ingle A.P., Yadav A.P., Rai M.K., Fabrication of silver nanoparticles by Phoma glomerata and its combined effect against Escherichia coli, Pseudomonas aeruginosa and Staphylococcus aureus. Lett. Appl. Microbiol., 2009, 48, 173-179.

[11] Ahmadi O., Jafarizadeh-Malmiri H., Jodeiri N., Eco-friendly microwave-enhanced green synthesis of silver nanoparticles using Aloe vera leaf extract and their physico-chemical and antibacterial studies. Green Process. Synth., 2018, 7, 231-240.

[12] Eskandari-Nojehdehi M., Jafarizadeh-Malmiri H., Jafarizad A., Microwave accelerated green synthesis of gold nanoparticles using gum Arabic and their physico-chemical properties assessments. Z. Phys. Chem., 2018, 232, 325-343.

[13] Fardsadegh B., Vaghari H., Mohammad-Jafari R., Najian Y., Jafarizadeh-Malmiri H., Biosynthesis, characterization and antimicrobial activities assessment of fabricated selenium nanoparticles using Pelargonium zonale leaf extract. Green Process. Synth., 2019, 8, 191-198.

[14] Aftab S., Shah A., Nadhman A., Kurbanoglu S., Ozkan S.A., Dionysiou D.D., et al., Nanomedicine: An effective tool in cancer therapy. Int. J. Pharm., 2018, 540, 132-149.

[15] Shah R.K., Haider A., Das L., Extracellular synthesis of ag nanoparticles using Escherichia coli and their antimicrobial efficacy. Int. J. Phatm. Biol. Sci., 2017, 7, 78-83.

[16] Nanda A., Saravanan M., Biosynthesis of silver nanoparticles from Staphylococcus aureus and its antimicrobial activity against MRSA and MRSE. Nanomed. Nanotechnol. Biol. Med., 2009, 5, 452-456. 